\title{
Centaurus : A Framework for Intelligent Services in a Mobile Environment
}

\author{
Lalana Kagal, Vlad Korolev, Harry Chen, Anupam Joshi, Timothy Finin \\ Computer Science and Electrical Engineering \\ University of Maryland Baltimore County \\ email : \{lkagal1,vkorol1,hchen4,ajoshi,finin $\} @$ cs.umbc.edu
}

\begin{abstract}
In an age where wirelessly networked appliances and devices are becoming commonplace, there is a necessity for connecting them to work together for a mobile user. The design outlined in this paper provides an infrastructure and communication protocol for providing 'smart' services to these mobile devices. This flexible framework allows any medium to be used for communication between the system and the portable device, including infra-red, and BlueTooth. Using Extensible Markup Language for information passing, gives the system a uniform and easily adaptable interface. We explain our trade-offs in implementation and through experiments we show that the design is feasible and that it indeed provides a flexible structure for providing services. Centaurus provides a uniform infrastructure for heterogeneous services, both hardware and software services, to be made available to the users everywhere where they are needed.
\end{abstract}

\section{Introduction}

As the world moves towards greater automation in homes and offices, we enter the realm of 'SmartHomes' and 'SmartOffices' controlled by sensors and/or portable devices, where not only has mobility been incorporated, but where intelligence has become an inherent part of providing services. Now-a-days, we see a lot of 'intelligent' services that use some kind of logical reasoning to provide better and more relevant support to individual users. These devices and services will have to be integrated seamlessly into the environment that the user is familiar with and provide a

This research was supported in part by the DARPA DAML program under contract F30602-97-1-0215, by National Science foundation grants NSF IIS-9875433 and NSF CCR-0070802 and through a contract from Aether Systems Incorporated. In Proceedings of International Workshop on Smart Appliances and Wearable Computing (IWSAWC), at the The 21st International Conference on Distributed Computing Systems (ICDCS-21) April 16-19, 2001. uniform interface to any device that the user might want to use.

Our goal is to provide an infrastructure and communication protocol for wireless services, that minimizes the load on the portable device. While within a confined space, the Client can access the services provided by the nearest Centaurus System (CS) via some short-range communication. The CS is responsible for maintaining a list of services available, and executing them on behalf of any Client that requests them. This minimizes the resource consumption on the Client and also avoids having the services installed on each Client that wishes to use them, which is a blessing for most resource-poor mobile clients.

We also expect all Services to communicate via Extensible Markup Language (XML). We found this W3C Standard [3] to be very useful in defining ontologies and describing properties and interfaces of Services. As this is already being widely used, we think that it will help in integrating Centaurus with already existing systems. The information flowing in the system is strictly in the form of CCML (Centaurus Capability Markup Language) which is built on top of XML.

To verify the feasibility of our infrastructure, we will use IR [8] for communication between the Client and the CS in our first stage of the development. One of the main drawbacks is the limitation of the infrared architecture. However, we believe that the simplicity and the affordability of the infrared devices can overcome these limitations. We would like to emphasize that any other medium could be used for communication including BlueTooth; all we provide is the framework.

This paper is organized as follows: Section II discusses other technologies. In Section III, the design and modeling issues are covered with the actual implementation being detailed in Section IV. The communication protocol is briefly illustrated in Section V. The results of the experiments are described in Section VI, we discuss some of our future research areas in Section VII and Section VIII concludes the paper. 


\section{Related Work}

In the last couple of years, a number of technologies have emerged that deal with 'Smart' Homes and Offices. Among them are the Berkeley Ninja Project [1], the Portolano project[16] from the University of Washington, Stanford's Interactive Workspaces Project [18], and Berkeley's Document-based Framework for Internet Application Control [10].

The team at Stanford has developed hardware and software testbeds that include large display devices as well as personal mobile computing devices such as laptops and PDAs connected through a wireless LAN. They are creating an infrastructure for multiple users to communicate with multiple devices with the ability to move work between different devices.

University of Washington's Portolano project is in the early stages and is involved in 'invisible computing' a term invented by Donald Norman [17]to describe ubiquitous computing, where devices supporting distributed services blend into the user's environment and become practically 'invisible'. The user would invoke these services not just by input but also through augmenting forms of interfacing like user movement, proximity of devices, identification tags, etc.

The Ninja project tries to link different services, through a range of devices ranging from PCs to cell phones and Personal Digital Assistants [1]. It has incorporated intelligence into the infrastructure and has the ability to adapt the content to a specific device.

Some differences lie in the implementation of the application, and the security infrastructure [2]. Currently we are working on the security aspect of the framework. We are using a system which combines Distributed Trust [20,21] and Kerberos [12,13]. It consists of a ticket granting server, that issues time-bound signed tickets for each mobile device. Ninja tends to concentrate on Web-based Services, whereas our system is able to support Services based on any platform, as long they can communicate with either the Service Manager through sockets, or one of the Communication Managers through the native protocol and possess the ability to process Centaurus Capability Markup Language(CCML) messages (this is discussed in Section 3.4). We also do not distinguish between hardware and software Services, allowing the user to use either in the same way. Unlike the Ninja project, Centaurus infrastructure delegates the state management to the Services themselves with the Service Manager serving as the cache. The advantage of such approach is the decreased complexity of distributed state management and increased fault tolerance. Even in the event of Service Manager going down, the state information is still preserved, and it will be uploaded back to the Service Manager after it comes back up. This happens be- cause the Services send regular status updates to the Service Manager. Since all of the communication between Services and Clients in the Centaurus project are done with the use of XML, there is no need for complicated Operators and Paths used by the Ninja project to convert between different data representations.

Though both the Ninja project and Centaurus are aimed at providing a uniform infrastructure for a multitude of devices to use heterogeneous services, Centaurus is more applicable for 'SmartHomes' and 'SmartOffices' because of its independence of any kind of specific communication infrastructure; so it could be easily implemented in the wide range of environments. In addition, Centaurus architecture is less prone to the failures of its components because of the use of multiple communication modules and automatic state recovery in the event of the Service Manager failure. When a Service Manager fails, it will be able to recover its state when the Services and Clients send it status updates (described in the Implementation section).

\section{Design}

A 'SmartRoom' is equipped with a Centaurus Communication Manager, which continuously broadcasts, through some medium, a client application. A person with a portable device who enters the room for the first time is given the option to install the software. Once the application is installed, it continuously reads the updated list of services. The person is able to choose a service, select a function, fill in the related options and execute the function. These services may be provided by Centaurus systems other than the one the portable device is connected to.

\subsection{Centaurus Communication Protocol (CComm)}

Centaurus protocol is used to communicate with mobile clients and services. The Centaurus protocol consists of Centaurus Level1 protocol and Centaurus Level2 protocols. Centaurus Levell Protocol is used as a glue between some existing communication architecture such as IrDA stack, BlueTooth, or TCP/IP and the generic Centaurus Level2 protocol. The Centaurus Level1 protocol handles connection and disconnection issues, identification and authentication of the clients and interaction with architecture specific protocols such as IrLAP and IrLMP or BlueTooth. The Centaurus Level 2 protocol handles transmission of the XML messages, time synchronization, message fragmentation and re-assembly. The Centaurus Level2 protocol is designed to be insensitive to disconnections, handle multiple clients, provide minimal turnaround times and be easily portable. In fact in the current implementation all communication managers and client communication modules use the exactly the same codebase. 


\subsection{Components}

There are four main components in a Centaurus System; the Service Managers, the Services, the Communication Managers, and the Clients. The Communication Managers handle all the communication with the Centaurus Client. The Communication Manager could implementing a number of different protocols by having different CComm modules, for example, one that handles IR, another that works with BlueTooth, one that works with HTTP to provide a web interface etc. The Service Managers are the controllers of the system that co-ordinate the message passing protocol between Clients and Services. The Services are objects that offer certain services to the Centaurus Client. At the present moment the Services contain information to enable them to locate the closet Service Manager and register themselves with it. Once registered, the Services can be requested by any Client talking to any Communication Manager. The Centaurus Client provides a user interface for accessing and executing Services. Figure 1 shows the different components and the relationships between them.

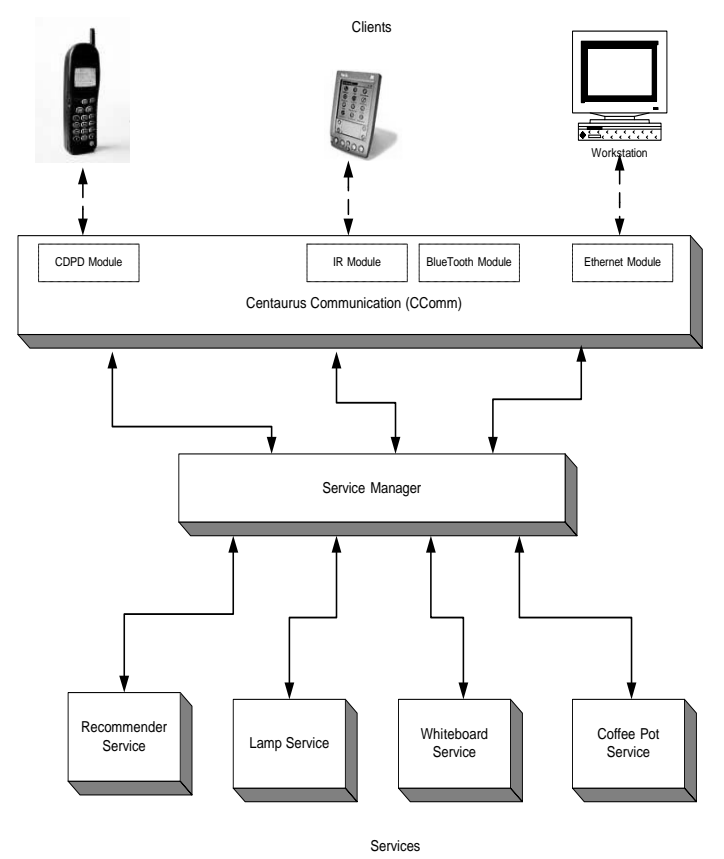

Figure 1. Centaurus Components

Service Manager. The Service Manager(SM) acts as a mediator between the Services and the Client. This is disregarding the fact that the Client sends the information to its Communication Manager that forwards it to the Service Manager. When a Service starts up, it has to register with the Service Manager, sending its CCML file. This file contains its name, id and the interfaces it implements. When a new Client comes along, the Service Manager sends it a ServiceList Object. This ServiceList object changes dynamically, according to the services registered with the Service Manager. So the Client always has the updated list of services. The Client can select a service, which causes the Service Manager to send the CCML file for the service. The Service Manager then updates its database to reflect that the specific Client is interested in the requested Service. Whenever the Service Manager gets a status update of the Service, it will send it to all interested Clients. The Client will continue to receive status reports from the Service, until it de-registers itself. The Client sends the new CCML file to the Service Manager, after invoking the interfaces of the Service. On receiving this CCML, the Service Manager validates the Client and the CCML. If the Service is still available, the Service Manager sends the CCML to it, otherwise it is queued for a for sometime. Once this timeout expires, an error is returned to the Client. The SM is also responsible for service discovery and leasing. It allows Services to register for a certain amount of time. If it does not receive any status update within that time, the registration is deleted. The SM implements an intelligent lookup for Services, enabling the Clients to search for Services that provide a certain kind of or related function.

Communication Manager. This is responsible for the communication between the Client and the Centaurus system. As mentioned earlier, the system can have a Communication Manager containing different CComm modules, one for every type of communication it wishes to implement. The Communication Manager talks via a certain socket to the Service Manager. This is allow Communication Managers and Service Managers to be on different system. When the Communication Manager receives information from a Client, it sends this information directly to the Service Manager through the socket. When it receives data from a Service Manager, it validates the data and looks at the header to decide which Client to send it to.

Services. A Service performs a certain action on behalf of the Client. These Services could range from controlling a light switch or a coffee pot to controlling a printer or even a memo pad service, where Clients can leave messages for each other. Each Service registers with a Service Manager by sending its CCML file, along with its name, id and a brief description of its functionality. Every time its status changes, it informs the Service Manager. It accepts requests only from the Service Manager that it is registered with.

Clients. A Client is a special kind of Service and is treated as a Service. It has to respond to commands and regularly send status updates. A Client talks to the Communication Manager and registers itself with a Service Manager. This registration is similar to the registration of Services. On registration, it receives the ServiceList, which contains the current list of Services. The ServiceList is a Service 


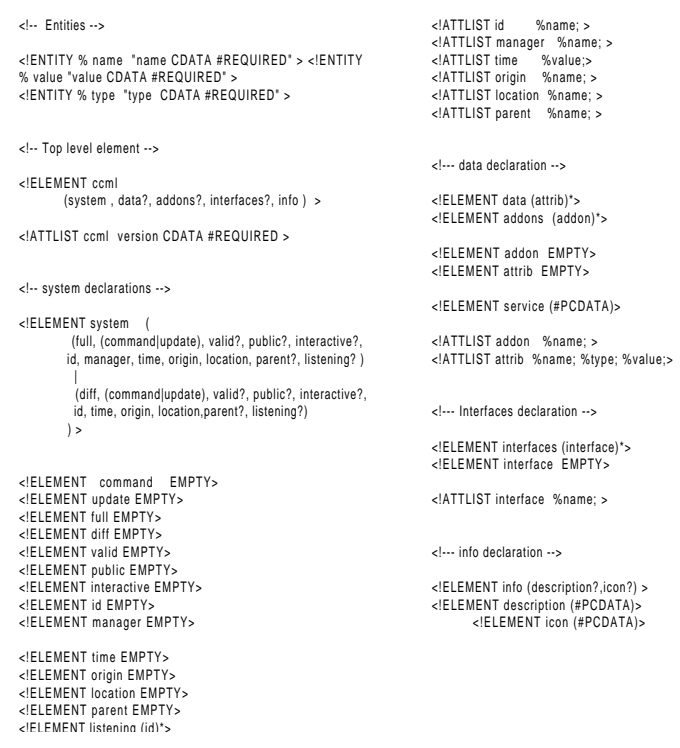

Figure 2. ccml.dtd

itself, and causes the Service Manager to send the list of Services, every time a new Service registers, or a Service de-registers.

By choosing a Service, the Client expresses interest in it. The Service Manager sends it the CCML file describing the Service. The Client can invoke the specified functions on the Service, by choosing one of its interfaces. After changing values of certain variables, specified in the CCML for the particular Service, it sends the file to the Service Manager to perform that action. It will receive status updates from all Services that it expresses interest in through the Service Manager, until it specifically informs the Service Manager that it no longer wants to receive these messages. Every time it wants to perform a certain action on a Service, it retrieves the current CCML file from its list, changes the appropriate values and returns the updated CCML to the Service Manager, which forwards it to the selected Service.

The way Centaurus is setup, Clients and the Service Managers only exchange XML messages. By providing XSL transforms, the XML messages can be rendered to fit the Client device. For example, if the Client wants to access a Lamp Control Service, the lamp can be turned on and off. This can be displayed as a true/false button on a PDA but on a cell phone, the the command could be spoken.

\subsection{Centaurus Capability Markup Language (CCML)}

The CCML is divided into 'system', 'data', 'addons', 'interfaces', and 'info', as shown in Figure 2. The 'system' portion contains the header information, the id, timestamp, origin, etc. There are two variables, 'update' or 'command'. An 'update' variable is used to inform other Centaurus components about status updates of Services and Clients, whereas the 'command' is only used by Clients to send a command to a certain Service. The system also contains the listening section for a Service or Client. It specifies all the Services that a Service or Client is interested in. Using the 'addons' section, we can add a related Service to another Service, for example, add an Alarm Clock Service to a Lamp-Control Service. We are not currently using this section. All information regarding the variables and their types are contained in the 'data' section. The CCML for a Client always has one or more 'actions' in its data section that a Service Manager can invoke on it. This is used by the SM to change the state of the device.

The following are the actions that are conveyed in the CCML

- AddService : When this action is set, the Client adds the value of this variable to its InterestList; i.e. the list of services that it is interested in.

- RemoveService : This is set by the Service Manager, if the Service that the Client is interested in, is no longer available. It causes the Client to stop listening or using the Service and remove the Service from its InterestList.

The 'interface' section contains information about the interfaces that the object (Service/Client) implements. Other details like the description, and icon for representation are in the 'info' section.

\subsection{Security}

Our security infrastructure combines certain functionality of Kerberos [12,13] system with Distributed Trust $[20,21]$. It consists of a ticket granting server (TS). Each Service Manager has to register with the TS. A mobile device wanting to use Centaurus, has to collect the appropriate ticket from the TS. The ticket is a set of rules specifying the rights of the device and is signed with the TS's private key. To access a Service on a certain Service Manager, the Client sends its CCML and the ticket. The Service Manager checks that the ticket is valid and from the TS and then allows the Client to register and use the appropriate Services.

\section{Implementation}

The previous section outlines our overall design, but to facilitate the implementation, we had to make some assumptions and sacrifice some of the features and flexibility. These assumptions in no way compromise the design or results, they only helped in quicker implementation.

To verify the feasibility of our infrastructure, we use IR [8] for communication between the Client and the Communication Manager in the first stage of the development. 
Though IrDa specifications say that IR transceivers have a 15 degree half-angle of view, we have observed that the PDA etc. exhibit at least 60 degrees. We have a single Communication Manager that uses IR. The IR Communication Manager carries out the Client discovery. Once discovered, the Client is polled regularly for information. This polling completely eliminates the problem with collision, that occurs in a client push method, when more than one Client sends information at the same time. We also have a single Service Manager and two Services for testing. We assume that the client application is installed on the PDA before it enters the 'SmartRoom'. Communication between any two components in the Centaurus System is done via sockets. The Service Manager and the IR Base Manager have two dedicated sockets each, one for listening and one for sending information. As the Service Manager and the IR Communication Manager are at the heart of all communication, we wanted to speed up this process. By giving them a dedicated socket for each type of communication, we reduced the time spent in the creation of a new socket for each connection. Each Service also has a socket for information from the Service Manager, which is sent to the Service Manager during the registration process. The Service Manager listens to a certain socket for receiving CCML from all the Services. All these sockets are predefined in the Properties file for each component. The information flowing in the system is strictly in the form of CCML (Centaurus Capability Markup Language).

The Service Manager and the Services have been implemented in Java, whereas we chose C for the IR Communication Manager and the Client, for increased efficiency in resource management. We found that most of the service discovery architectures are implemented in Java, like Jini and E-Speak. So, if we decide to move to another service discovery system, integration will be relatively easy as the Service Manager and Services are already in Java.

\subsection{Services}

We have developed one hardware related service for controlling a lamp and one software service for playing MP3 files. There is another Service, ServiceList, that is an inherent part of the protocol, and is used for providing an updated list of services to the Client.

We have implemented a Service class and ServiceInterface class, that handle validation of the CCML, the registering of the Service with the Service Manager and the sending of the updates. All Services implemented in Java should, for conformity, extend the Service class, and implement the ServiceInterface class. The ServiceInterface class contains a commandHandler function that has to be implemented by every Service that implements the interface. This is the function that handles changes to the CCML file of the
Service. A Java Service need only implement a constructor and this commandHandler to be integrated into a Centaurus system.

As mentioned earlier, the Centaurus system also handles non-Java Services as long they can use CCML and either communicate via sockets with the Service Manager or with a Communication Manager through some native protocol.

- ServiceList. Each time, a Service registers or is no longer available, the ServiceList triggers the Service Manager to send the updated list of Services to all the Clients. This does not use the Service class or the ServiceInterface class. It is contained completely in the Service Manager. It is a special Service because it is handled in same way as other Services are, but within the Service Manager itself.

- Lamp-Control. Using X10 [11] devices and FireCracker [9], we were able to control a lamp in the room. We can extend this to control any device because X10 is a power-line carrier protocol that allows compatible devices to communicate with each other via the existing $110 \mathrm{~V}$ wiring. FireCracker is a Java class that allows a computer to communicate with the X10 device. The Service constructor makes sure that the X10 device works. The commandHandler function looks for the value of the interfaces. If the 'Powered' interface has a value that is different from the status of the Power variable, then the commandHandler proceeds, otherwise the command is discarded. If the value is true, the lamp is set on, otherwise the lamp is set off. The CCML file is changed and an 'update' is sent to the Service Manager, which propagates all the way back to all the Clients that are interested in the lamp service.

- MP3-Player. We are using a popular MP3 player for Unix, mpg123 [14], that has a Java wrapper around it to allow us to plug it into the rest of the system. The constructor for the Service, reads all the .mp3 files from a specified directory and creates its CCML files. It has a number of CCML interfaces, one for each song it can play. The commandHandler function checks the CCML interface and reads the songs selected. These songs are checked against the current list of songs. If they are valid, they are fed into mpg123 [14]. The new CCML file is created and sent to the Service Manager.

\subsection{Functions}

Registration. Both Services and Clients have to register with the Centaurus system to be visible. A Service on starting up, reads its properties file and retrieves the Service Manager's port number. After creating its CCML file, it sends its CCML and the port number that it is listening to, to the Service Manager's Service port. The Service Manager validates the CCML and adds the Service to its Services list.

When a PDA enters the 'SmartRoom', we assume that it has the Client application installed on it, as mentioned in the beginning of this section. It is eventually discovered by the IR Communication Manager, and carries out the IR level protocol. Then it sends its pre-defined CCML file. 
The IR Communication Manager sends this to the Service Manager's port. The Service Manager after validating the CCML checks if the Client already exists in its Clients list. If it does, then the Service Manager updates its list, otherwise it adds the Client to its Clients list. The Service Manager, sets the ServiceList action and sends the CCML back to the Client.

Requesting a Service. When a Client receives the list of Services, it displays this list for the user. The user can select a Service to use. The Client then creates a command for the ServiceList. It changes the data portion of the ServiceList, with the value of the Service selected as 'true'. This is sent back to the Service Manager. As it is a command for the ServiceList, which is part of the Service Manager, the Service Manager handles it. From the system section, the Service Manager retrieves the name of the Client and checks the data section for the Services. It then retrieves the latest CCML for the Client from its Clients list and creates a command for the Client. It sets the AddService action to the Services selected and sends the CCML back to the Client. The Client processes this CCML as it would any AddService action by adding the Service to its listening section. It also adds the Service to its InterestList. When the Client is next polled it sends its updated CCML. The Service Manager reads the list of Services that the Client is listening to, and picks out the new Services, ones that the Client was not previously listening to. It sends their CCML to the Client via the IR Communication Manager. It then, adds the new Service-Client pair to its Service-Client list. Once the Client gets the CCML of the Service, it displays it for the user. The user can use the interfaces to perform actions. The Client modifies the Service's CCML to make a command, sets the new values and sends when polled. The Service Manager realizes that it is a command and sends it to the appropriate Service. The Service carries out the command and sends the update to the Service Manager.

Status Update. If a Service Manager receives an update from a Service, it checks its Service-Client list for all the Clients interested in this Service. It sends the updated CCML to these Clients. When a Service Manager receives an update from a Client, it carries out certain functions on it. It checks the listening section and retrieves the list of Services that the Client is listening to. It picks out the new Services, ones that the Client was not previously listening to. It sends their CCML to the Client via the IR Communication Manager. Then for each new Service, it adds a new Service-Client pair to its Service-Client list.

\section{Centaurus Communication Protocol}

CComm is the layer under Communication Manager, and provides communication with the mobile devices.

The Centaurus Levell protocol is running on top of
IRDA's IrLAP and IrLMP protocols. [8] IrLAP is a low level protocol that provides device to device connection for reliable data transfer, device discover procedures and hidden node handling. IrLMP works on top of IrLAP and handles multiplexing of the IrLAP layer and multiple channels above an IrLAP connection as well as protocol and service discovery via the Information Access Service (IAS). The Centaurus Level1 protocol provides the glue between IRDA protocols (IRLAP and IRLMP) and Centaurs level 2 protocol.

Centaurus level 2 protocol is based on passing short command messages between the client and the server. These command messages are used to establish the session ( HELO, HELORSP), synchronize clocks (POLL), find out which objects are available for transmission(OBJ,POLL, NONE), handle flow control (PROCEED, DONE, ACK) and do actual data transmission (PK).

The client and server use exactly the same code for both level 1 and level 2 protocols implementation, the only difference is the actions that are performed by the level 1 protocol to establish connection, and handling of $\mathrm{OBJ}$ and $\mathrm{NONE}$ messages by the level 2 protocol stack.

The level 1 protocol differences are the following. On the server side after connection is established and authentication is performed the level 1 protocol sends 'HELO' message and starts up the level 2 protocol. On the client side the level 1 protocol waits for the initial HELO message, after it receives this message it replies back with HELORSP message and starts up the level 2 protocol as well.

Although most of the messages in the protocol are just plain text strings few messages deserve further explanation. The POLL message, if transmitted from the server, is followed by the current value of the server clock. The OBJ message is followed by the object name, its time stamp and its size. The PK message is followed by the actual data payload. The PROCEED message is followed by the received packets bitmap, when a packet is received by the receiver, it marks the bit corresponding to this packet number in the received packets bitmap, when all of the bits in a bitmap are marked the message is said to be received and receiver sends ACK message back to the sender. If for some reason disconnection occurs before the whole message is sent, during the next session the receiver sends the received packets bitmap to the sender and the sender either proceeds to send the remaining packets or discards the whole object if newer version is available.

\section{Results}

While testing, we had 3 PDAs in the room communicating with the Centaurus system. Each PDA requested one or two Services, and executed them. We even tried simultaneous execution of Services by the PDAs. These were 
automatically handled by the IR Communication Manager, which polled the PDAs at regular intervals, so only one request was sent to Service at a time. We believe that the testing was successful as we were able to execute both the Services by any of the PDAs.

\section{Future Work}

We are working on the security portion of our framework, and are implementing it at 2 levels, at the Service Manager who checks the Client's ticket and at the Services themselves. The Services can validate the ticket and decide whether the Client should be allowed access.

We are also working on a Recommender Service. Instead of returning a list of all possible services that are available to a Client, this service recommends a list of services that might be in the interest of the Client based on the existing environment context. For example, the system returns a coffee-maker control service during the morning to the user, and in the evening it returns a light control service to the user. It may also notice that the user generally wants to listen to to the same list of songs and provide the list as soon as the user steps into the room.

We would like to arrange the Service Managers into a hierarchy so that the Services could connect to the closest Service Manager, and the location of a Service Manager need not be coded into the Services. This will also allow the Services to be shared across the Service Managers, so a user could enter one room and use the printer in another room by using the printer Service on the Service Manager in the other room.

\section{Conclusion}

We have successfully developed the first version of Centaurus. We believe that by providing a uniform infrastructure using XML-encoded data exchange we have shown that it is appropriate and effective for deploying services in an indoor environment. The first stage development, including the Service Manager, IR Communication Manager, MP3 player services, Lamp services etc. has verified that our vision is definitely feasible. Although, our project is far from complete, we believe that now that the framework is in place, adding attractive interfaces for the portable devices, creating new services, and enabling more intelligent brokering of Services will follow easily. We believe that we have crossed all the major hurdles, and completing the remaining portion will be pretty straightforward.

\section{References}

[1] The Ninja Project http://ninja.cs.berkeley.edu/
[2] Steven E. Czerwinski, Ben Y. Zhao, Todd D. Hodes, Anthony D. Joseph, and Randy H. Katz, "An Architecture for a Secure Service Discovery Service" Fifth Annual International Conference on Mobile Computing and Networks (MobiCom '99)

[3] W3C Consortium http://www.w3.org/XML/

[4] The Official BlueTooth Website http://www.bluetooth.com/

[5] Internet Business Solutions : E-Speak http://www.e-speak.hp.com?qt=espeak/

[6] Jini Connection Technology Executive Overview http://www.sun.com/jini/

[7] Service Location Protocol http://www.svrloc.org/index.html

[8] Infrared Data Association http://www.irda.org

[9] FireCracker http://www.x10.com/welcome/firecracker/

[10] T. Hodes, R. H. Katz, "A Document-based Framework for Internet Application Control", Proceedings of the Second USENIX Symposium on Internet Technologies and Systems (USITS '99), October 1999

[11] X10 Devices http://www.x10.com/

[12] B. Clifford Neuman and Theodore Ts'o., "Kerberos: An Authentication Service for Computer Networks", IEEE Communications, September 1994

[13] John T. Kohl, B. Clifford Neuman, and Theodore Y. T'so, "The Evolution of the Kerberos Authentication System", Distributed Open Systems, IEEE Computer Society Press, 1994 ftp://athena-dist.mit.edu/pub/kerberos/

[14] Mpg123, MP3 Player for Linux/Unix Systems http://mpg123.org/

[15] Swedish Institute of Computer Science, SICStus Prolog http://www.sics.se/sicstus/docs/latest/html/sicstus.html

[16] University of Washington, Dept of Computer Science and Engineering, "Portolano: An Expedition into Invisible Computing" http://portolano.cs.washington.edu/

[17] "The Invisible Computer", D. Norman, MIT Press, 1998.

[18] Stanford Interactive Workspaces Project http://graphics.stanford.edu/projects/iwork/

[19] Monarch Project http://www.monarch.cs.cmu.edu/

[20] M.Blaze, J.Feigenbaum, J.Lacy, ”Decentralized Trust Management" ,IEEE Proceedings of the 17th Symposium on Security and Privacy, 1996

[21] M.Blaze, J.Feigenbaum, M.Stauss, "Compliance Checking in the Policy Maker Trust Management System", Proceedings of Financial Crypto'98, Lecture Notes in Computer Sciences vol.1465, Springer Berlin, 1998 